\title{
Urine Culture Testing in Community Nursing Homes: Gateway to Antibiotic Overprescribing
}

\author{
Philip D. Sloane, MD, MPH; ${ }^{1,2}$ Christine E. Kistler, MD, MASc; ${ }^{1,2}$ David Reed, PhD; ${ }^{2}$ David J. Weber, MD, MPH; ${ }^{3,4}$ \\ Kimberly Ward; ${ }^{2}$ Sheryl Zimmerman, $\mathrm{PhD}^{2,5}$
}

\begin{abstract}
ов јестіvе. To describe current practice around urine testing and identify factors leading to overtreatment of asymptomatic bacteriuria in community nursing homes (NHs).

DESIGN. Observational study of a stratified random sample of NH patients who had urine cultures ordered in NHs within a 1-month study period.
\end{abstract}

Setting. $31 \mathrm{NHs}$ in North Carolina.

PARTICIPANTS. $254 \mathrm{NH}$ residents who had a urine culture ordered within the 1-month study period.

MEтHODS. We conducted an NH record audit of clinical and laboratory information during the 2 days before and 7 days after a urine culture was ordered. We compared these results with the urine antibiogram from the $31 \mathrm{NHs}$.

RESULTS. Empirical treatment was started in 30\% of cases. When cultures were reported, previously untreated cases received antibiotics $89 \%$ of the time for colony counts of $\geq 100,000 \mathrm{CFU} / \mathrm{mL}$ and in $35 \%$ of cases with colony counts of $10,000-99,000 \mathrm{CFU} / \mathrm{mL}$. Due to the high rate of prescribing when culture results returned, $74 \%$ of these patients ultimately received a full course of antibiotics. Treated and untreated patients did not significantly differ in temperature, frequency of urinary signs and symptoms, or presence of Loeb criteria for antibiotic initiation. Factors most commonly associated with urine culture ordering were acute mental status changes $(32 \%)$; change in the urine color, odor, or sediment $(17 \%)$; and dysuria $(15 \%)$.

CONCLUSions. Urine cultures play a significant role in antibiotic overprescribing. Antibiotic stewardship efforts in NHs should include reduction in culture ordering for factors not associated with infection-related morbidity as well as more scrutiny of patient condition when results become available.

Infect Control Hosp Epidemiol 2017;38:524-531

All US nursing homes (NHs) are required to implement antibiotic stewardship programs by November $2017,{ }^{1}$ due to the increasing prevalence of multidrug-resistant organisms (MDROs) and research demonstrating that as many as 75\% of $\mathrm{NH}$ antibiotic prescriptions are unnecessary or are for the wrong drug, dose, or duration. ${ }^{2,3}$ A suspected urinary tract infection (UTI) is the most common reason $\mathrm{NH}$ antibiotics are prescribed, ${ }^{4,5}$ and UTI prescriptions are key contributors to the emergence of MDROs. ${ }^{6-8}$ Approximately half of these prescriptions are not for a UTI but for asymptomatic bacteriuria, a condition for which treatment does not improve health outcomes but does increase drug resistance and may lead to serious adverse events. ${ }^{10,11}$

Thus, an important component of $\mathrm{NH}$ antibiotic stewardship must be improved evaluation and diagnosis of UTIs.
Urine testing often drives prescribing; so a key component of antibiotic stewardship around NH UTIs must be the ordering and interpretation of urine tests. ${ }^{6,12}$ Evidence-based guidelines for urine testing have been developed; the most explicit are the 2008 update by the Infectious Diseases Society of America (IDSA), ${ }^{13}$ the consensus criteria of Loeb, et al, ${ }^{14,15}$ and the updated criteria of Genao and Buhr. ${ }^{16}$ All of these rely heavily on focal urinary tract symptoms as a prerequisite of testing.

Despite the high level of interest in changing $\mathrm{NH}$ practice around UTIs, little information exists about decision making in community-based NHs or the areas of practice that might be most amenable to change. To address this gap, we identified and reviewed 254 cases from 31 community NHs in which urine cultures were ordered by medical providers. Our goals were (1) to describe the decision-making process around urine

Affiliations: 1. Department of Family Medicine, School of Medicine, University of North Carolina, Chapel Hill, North Carolina; 2. Cecil G. Sheps Center for Health Services Research, University of North Carolina, Chapel Hill, North Carolina; 3. Division of Infectious Diseases, Department of Medicine, School of Medicine, University of North Carolina, Chapel Hill, North Carolina; 4. Department of Epidemiology, School of Public Health, University of North Carolina, Chapel Hill, North Carolina; 5. School of Social Work, University of North Carolina, Chapel Hill, North Carolina.

Received August 25, 2016; accepted December 6, 2016; electronically published January 31, 2017 DOI: $10.1017 /$ ice.2016.326 
testing, including documentation, prescribing decisions, and outcomes, and (2) to identify areas amenable to quality improvement efforts aimed at reducing unnecessary antibiotic prescribing.

\section{METHODS}

\section{Setting and Study Population}

As part of a research study regarding infection management and antibiotic stewardship in NHs, we enrolled 31 community-based NHs in North Carolina, and within each study $\mathrm{NH}$, we audited a sample of cases in which urine cultures had been ordered. To promote $\mathrm{NH}$ buy-in and thereby assure a more representative sample, $\mathrm{NHs}$ were approached through either a for-profit regional $\mathrm{NH}$ chain or a regional long-termcare medical group. A total of $35 \mathrm{NHs}$ were approached for participation; 4 refused. In each participating $\mathrm{NH}$, a team of geriatricians and research staff made a site visit between November 2014 and March 2015 to conduct medical record audits. From a list of all urine cultures reported in each $\mathrm{NH}$, the study team randomly selected up to 10 cases from the previous month; in NHs having fewer than 10 cases, all available cases were audited, yielding a total of 254 cases. Study methods were approved by the Institutional Review Board of the University of North Carolina at Chapel Hill.

\section{Measures}

For each case, medical and nursing records were systematically audited to record signs and symptoms during the 48 hours before a test was ordered, guided by the published literature on signs and symptoms associated with UTIs in older persons. ${ }^{13-18}$ The following data were also collected: the reason for the culture (if stated); urinalysis and culture results, including type and quantity of bacteria as well as antibiotic resistance patterns; antibiotic treatment decisions on the day the test was ordered and during the subsequent 5 days; signs and symptoms during days 2-5 after a test was ordered (ie, when a urine culture result would have become available); and whether or not the patient had an emergency department visit, hospitalization, or death during the 7 days after the culture was ordered. Because only 1 of the $31 \mathrm{NHs}$ performed on-site urine testing, dipstick and urinalysis results were not included in these analyses. To adjust for difference in the method of measuring temperature, we subtracted $1.35^{\circ} \mathrm{C}$ $\left(0.75^{\circ} \mathrm{F}\right)$ from rectal and tympanic readings and added $1.35^{\circ} \mathrm{C}$ $\left(0.75^{\circ} \mathrm{F}\right)$ to axillary readings to estimate an oral temperature equivalent. $^{19}$

To better estimate the concordance of empirical antibiotic selection with $\mathrm{NH}$-specific antibiotic resistance patterns, the study team gathered all urine culture reports for the 31 study NHs between December 1, 2014, and August 31, 2015, for a total of 2,985 cultures reported by 21 different laboratories. Because large samples are needed to construct stable antibiograms, these results are reported at the aggregate level and not for specific NHs.

\section{Statistical Analysis}

Analyses included descriptive statistics, a comparison of empirical antibiotic choices with aggregate data on sensitivity and resistance patterns of commonly cultured bacteria, and the calculation of the proportion of cases consistent with the Loeb criteria for initiation of antibiotics for presumed UTIs and the modified IDSA criteria for surveillance of UTIs. ${ }^{15,17}$ In applying the IDSA criteria, "constitutional criteria" other than fever were omitted because no NHs routinely assessed residents using the 28-point functional status index or the quantitative confusion assessment method, as required by these criteria. ${ }^{17}$ The statistical significance of differences in the proportion of residents treated with empirical antibiotics was tested using Pearson's $\chi^{2}$ test, and Fisher's exact test was used when any cell count was $\leq 10$.

\section{RES ULT S}

Of the 31 community NHs that participated in this study, $81 \%$ were for-profit facilities. The mean bed size was 113; the mean occupancy rate was $87 \%$; the mean work hours per resident for licensed nurses and certified nursing assistants, respectively, were 1.5 and 2.2; and the mean quality rating on Nursing Home Compare was 3.3. None of these values were statistically different from the mean of all NHs nationally. ${ }^{20}$

\section{Clinical Features and Treatment Decisions at the Time a Urine Culture Was Ordered}

Urine cultures were obtained using a variety of methods. More than one-third $(36 \%)$ of $\mathrm{NH}$ records did not document the method by which the culture was obtained; the remainder included $26 \%$ collected by straight (in-and-out) catheterization, $20 \%$ as voided specimens, and $19 \%$ from an indwelling device.

On the day of or before a urine culture was ordered, $32 \%$ of patients whose urine was cultured were reported to have an acute mental status change; $17 \%$ were reported to have a change in the urine color, odor, or sediment; $14 \%$ had dysuria; $14 \%$ had a body temperature documented at $\geq 37.2^{\circ} \mathrm{C}\left(99^{\circ} \mathrm{F}\right)$; and $74 \%$ lacked documentation of any urinary-tract-specific signs or symptoms. Only $22 \%$ of these patients met the Loeb criteria for ordering a urine culture (Table 1).

In almost one-third of cases (30\%), empirical treatment with antibiotics was started before the culture report was available. Only temperature elevation, increased urinary frequency, and change in urine appearance were significantly associated $(P \leq .05)$ with empirical antibiotic prescribing; a negative urine dipstick for nitrite and leukocyte esterase was significantly associated with a decision to withhold treatment. Treated cases were no more likely than untreated cases to meet 
тав

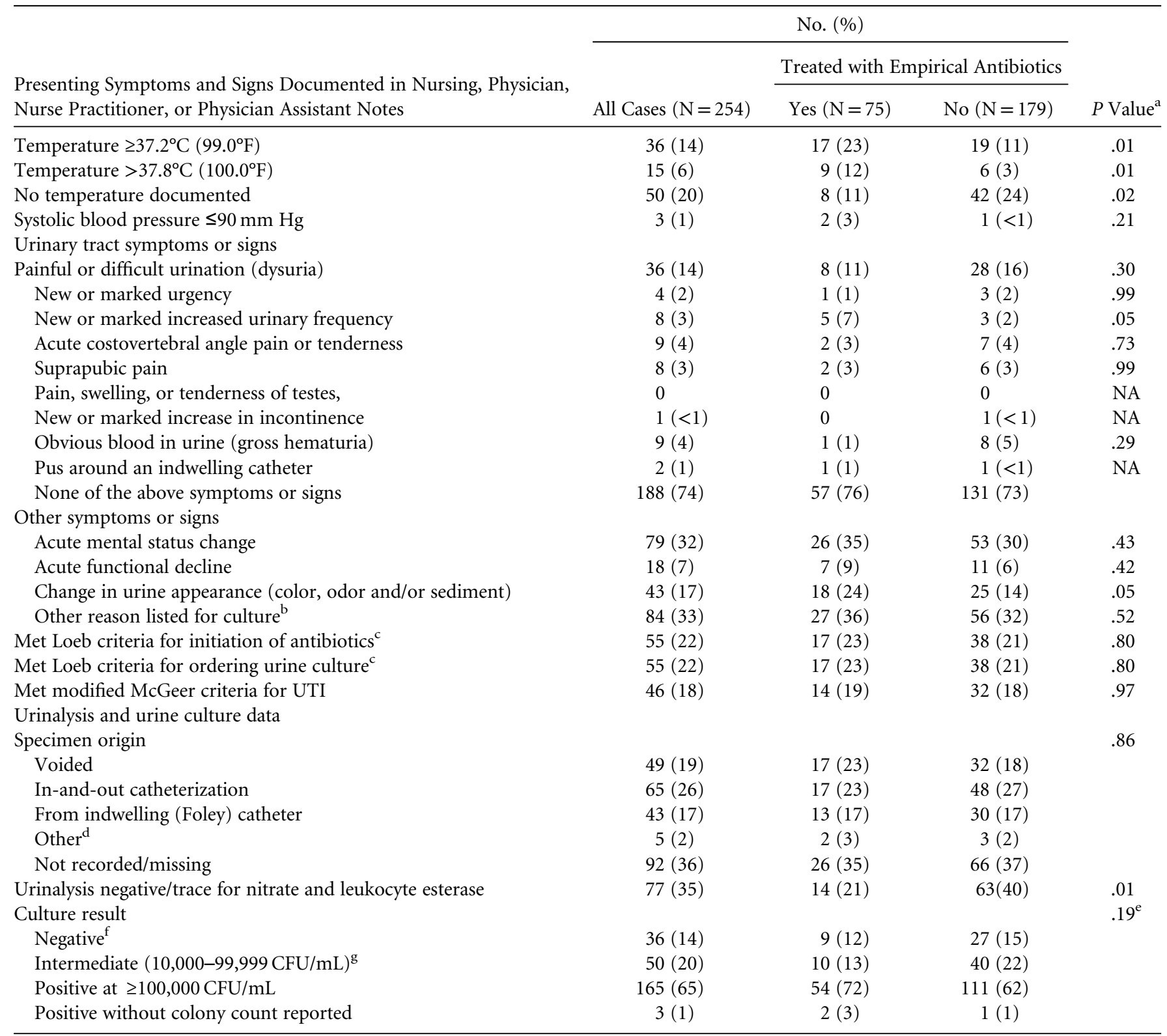

NOTE. NA, not appropriate; UTI, urinary tract infection; CFU, colony-forming units.

${ }^{a}$ Pearson $\chi^{2}$ test. Fisher's exact test used where any cell count is $\leq 10$.

'The most common "other" reasons documented for urine collection were fall and/or syncope (14.3\%), "history of" chronic or recurrent UTIs (9.5\%), "urinary retention" (9.5\%), agitation/behavior change $(8.3 \%)$, and increased white blood cell count (8.3\%).

${ }^{\mathrm{c}}$ Loeb 2005.

${ }^{\mathrm{d}}$ The 5 specimens collected using "other" methods included ileal conduit $(\mathrm{N}=1)$ and suprapubic catheter $(\mathrm{N}=4)$.

${ }^{\mathrm{e}} P$ value for 3 categories, with the 3 patients with an organism reported without a colony count excluded.

$\mathrm{f}_{32}$ cultures showed no growth and 4 cultures showed $<10,000 \mathrm{CFU} / \mathrm{mL}$.

gIncludes 22 "polymicrobial" with no organism reported.

the 2005 Loeb criteria for empirical initiation of antibiotics for presumed UTI in NH residents or the modified McGeer surveillance criteria for UTIs (Table 1).

In the 75 cases treated empirically with antibiotics, the most commonly prescribed agents were ciprofloxacin (31\%), trimethoprim-sulfamethoxazole (20\%), nitrofurantoin (11\%), ceftriaxone $(11 \%)$, and levofloxacin $(7 \%)$. When the most commonly prescribed antibiotics were compared with the antibiogram we constructed from 2,985 consecutive urine cultures from study NHs, high levels of resistance were 
table 2. Relationship Between the Antibiotics Most Commonly Prescribed Empirically for a Presumed Urinary Tract Infection and Resistance Patterns Among Positive Urine Cultures From 31 Nursing Homes ${ }^{a}$

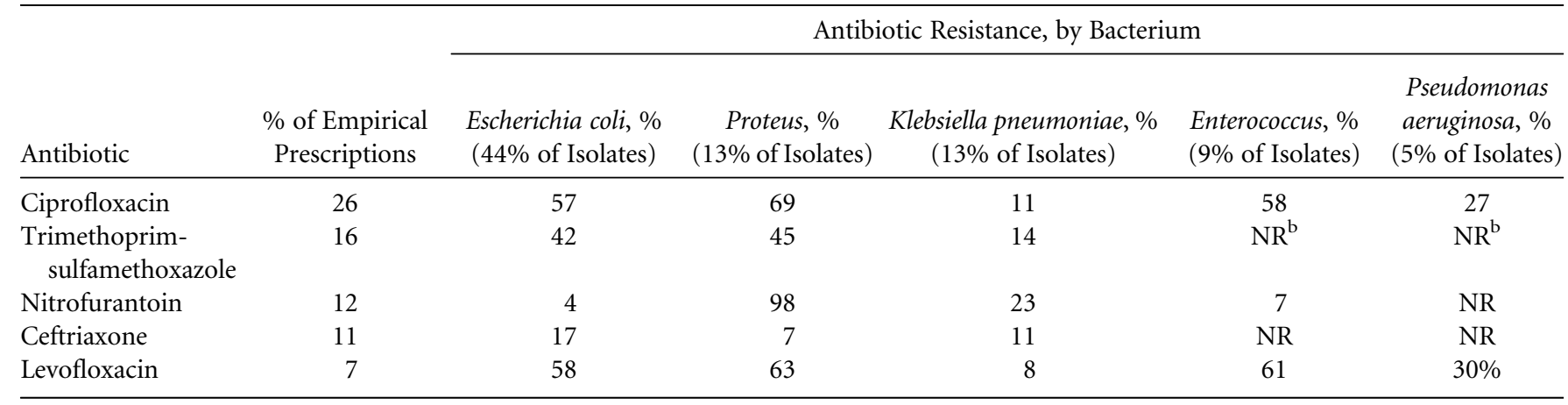

NOTE. NR, not reported.

${ }^{a}$ The study included 75 empirical antibiotic prescriptions. The antibiotic resistance antibiogram is based on 1,580 positive urine cultures (ie, $\geq 100,000 \mathrm{CFU} / \mathrm{mL}$ ) from 31 nursing homes.

${ }^{b}$ Not reported because resistance is known to be very high.

observed, with E. coli (the most common pathogen) being resistant to ciprofloxacin in $57 \%$ of cases and to trimethoprimsulfamethoxazole in $42 \%$ of cases (Table 2).

\section{Clinical Features and Treatment Decisions When a Urine Culture Became Available}

Results from reporting laboratories indicated that, of these 254 specimens, 36 (14\%) had no bacteria, 50 (20\%) had intermediate colony counts $(\leq 100,000 \mathrm{CFU} / \mathrm{mL}$ or polymicrobial results with no organism reported, ie, "contaminated" specimens), $165(65 \%)$ were positive with $\geq 1$ bacteria at $\geq 100,000 \mathrm{CFU} / \mathrm{mL}$, and $3(1 \%)$ had an organism reported but no colony count report. Of the positive cultures, the majority (84\%) yielded a single organism, $15 \%$ yielded 2 bacteria, and $1 \%$ reported 3 bacteria (Table 1).

Our evaluation of prescribing decisions made during days 2-5 after a culture had been ordered (and the result would have been available in the $\mathrm{NH}$ ) indicates that a positive culture in a patient who was not on antibiotics $(\mathrm{N}=111$ cases) led to initiation of an antibiotic in $89 \%$ of cases. Treated patients with positive urine cultures did not, during the days when the culture results became available, differ significantly from untreated patients in temperature, frequency of urinary signs and symptoms, or presence of Loeb criteria for initiating antibiotics, and only a small minority (13\%) met the modified McGeer criteria for having a UTI (Table 3).

Among the 9 patients who had been initially prescribed antibiotics and had negative urine culture results, none had their antibiotic stopped, and the prescription was changed for 1 patient despite a colony count $<10,000 \mathrm{CFU} / \mathrm{mL}$. Among the 10 patients who were initially prescribed antibiotics whose colony counts were intermediate, antibiotics were stopped in only 2 . Among the 67 patients who had not been prescribed antibiotics and who had cultures with colony counts $<100,000 \mathrm{CFU} / \mathrm{mL}, 16(24 \%)$ were started on antibiotics.
Of these, 13 cultures had bacteria at colony counts between 10,000 and $99,000 \mathrm{CFU} / \mathrm{mL}$, while 2 had polymicrobial results (without sensitivities reported), and only 1 was completely devoid of bacteria. Overall, 191 (75\%) urine cultures were associated with at least 1 antibiotic prescription, and 189 (74\%) of all subjects completed a full course of antibiotics (Figure 1).

\section{Adverse Patient Events}

During the 7 days after the 254 urine cultures were ordered, 2 study participants died and 2 others were hospitalized with a diagnosis of "sepsis" or "bacteremia." Of the 2 deaths, 1 patient had a positive urine culture for Pseudomonas that had been ordered the day before she died, at which time she was on hospice care, was afebrile, and was experiencing functional decline. The other had recent hip replacement surgery, a Foley catheter, and a culture that reported $90,000 \mathrm{CFU} / \mathrm{mL}$ of Staphylococcus spp.; 7 days after the urine culture was ordered her incision dehisced and drained pus, after which she was transferred to the hospital and subsequently died. Of the 2 patients who were hospitalized and returned to the $\mathrm{NH}$ with discharge diagnoses that included "sepsis" both had negative urine cultures at the $\mathrm{NH}$.

\section{DISCUSSION}

In this study of 254 urine cultures ordered in 31 community NHs, we found that, in the face of little documentation of acute UTI, the act of ordering a culture led to an antibiotic prescription in the vast majority of cases. Empirical antibiotics were started in $30 \%$ of patients, often with antibiotics that have a moderate-to-low likelihood of being effective due to antibiotic resistance patterns of common urinary pathogens in these NHs, thereby risking alteration in normal bowel and other flora without treating the presumed pathogen. ${ }^{21}$ When urine culture reports became available, although most patients 
ta вLE 3. Symptoms and Treatment Decisions During Days 2-5 After a Urine Culture among 178 Nursing Home Residents Who Were Not Treated Empirically Before the Culture Result Was Available ${ }^{\mathrm{a}}$

\begin{tabular}{|c|c|c|c|c|}
\hline $\begin{array}{l}\text { Presenting Symptoms/Signs and Treatment Documented in Nursing, } \\
\text { Physician, Nurse Practitioner, or Physician Assistant Notes or Orders }\end{array}$ & $\begin{array}{l}\text { All Cases, No. } \\
(\%)(\mathrm{N}=178)\end{array}$ & \multicolumn{3}{|c|}{ Culture Result, No. $(\%)^{\mathrm{b}}$} \\
\hline Temperature $>37.8^{\circ} \mathrm{C}\left(100.0^{\circ} \mathrm{F}\right)$ & $2(1)$ & $1(1)$ & 0 & .99 \\
\hline No temperature documented & $86(48)$ & $33(30)$ & $53(79)$ & $<.001$ \\
\hline Painful or difficult urination (dysuria) & $13(7)$ & $10(9)$ & $3(5)$ & .38 \\
\hline New or marked urgency & $2(1)$ & $2(2)$ & 0 & .53 \\
\hline New or marked increased urinary frequency & $4(2)$ & $3(3)$ & $1(2)$ & .66 \\
\hline Acute costovertebral angle pain or tenderness & 0 & 0 & 0 & $\ldots$ \\
\hline Suprapubic pain & $1(1)$ & $1(1)$ & 0 & .99 \\
\hline Pain, swelling, or tenderness of testes, epididymis, or prostate & 0 & 0 & 0 & $\ldots$ \\
\hline \multicolumn{5}{|l|}{ Other symptoms or signs } \\
\hline Acute mental status change & $12(7)$ & $8(7)$ & $4(6)$ & .77 \\
\hline Acute functional decline & $5(3)$ & $5(5)$ & 0 & .16 \\
\hline Change in urine appearance (color, odor and/or sediment) & $6(3)$ & $5(5)$ & $1(2)$ & .41 \\
\hline Other reason listed for culture & $7(4)$ & $5(5)$ & $2(3)$ & .71 \\
\hline Met Loeb criteria for initiation of antibiotics & $16(9)$ & $13(12)$ & $3(5)$ & .11 \\
\hline Met Loeb criteria for ordering urine culture & $16(9)$ & $13(12)$ & $3(5)$ & .11 \\
\hline Met modified McGeer criteria for UTI & $15(8)$ & $14(13)$ & $1(2)$ & .01 \\
\hline Treatment decisions: New antibiotic prescription made & $115(65)$ & $99(89)$ & $16(24)^{\mathrm{d}}$ & $<.001$ \\
\hline No new antibiotic prescription made & $63(35)$ & $12(11)$ & $51(76)$ & $<.001$ \\
\hline
\end{tabular}

NOTE. UTI, urinary tract infection; CFU, colony-forming units.

${ }^{a}$ Among the 179 patients who did not initially receive antibiotics, 1 patient had no colony count reported and was excluded.

${ }^{\mathrm{b}} \mathrm{A}$ positive culture result was defined as having a colony count $\geq 100,000 \mathrm{CFU} / \mathrm{mL}$; an intermediate result as between 10,000 and $99,000 \mathrm{CFU} / \mathrm{mL}$ or polymicrobial with no organism identified; and a negative culture as no growth or $<10,000 \mathrm{CFU} / \mathrm{mL}$.

${ }^{c}$ Pearson $\chi^{2}$ test (Fisher's exact test used where any cell count is $\leq 10$ ).

${ }^{\mathrm{d}}$ Among these cases, $13 \mathrm{had}$ an organism reported at $<100,000 \mathrm{CFU} / \mathrm{cm}^{3} ; 2$ were reported as "polymicrobial"; and 1 reported no bacteria.

lacked constitutional or urinary-tract-specific symptoms, the presence of bacteria usually led to an antibiotic prescription in persons who had not been treated empirically, and a negative culture rarely led to the discontinuation of empirically prescribed antibiotics. As a result, $74 \%$ of urine cultures were associated with completion of a course of antibiotics.

Why many of these cultures were ordered is unclear. Most patients lacked documentation of either a temperature $\geq 37.2^{\circ} \mathrm{C}\left(99.0^{\circ} \mathrm{F}\right)(86 \%)$ or of any urinary tract signs or symptoms ( $74 \%$ ), and only $22 \%$ satisfied criteria for ordering a urine culture that were developed to reduce unnecessary urine cultures. ${ }^{22}$ The result was overtreatment of asymptomatic bacteriuria, a common $\mathrm{NH}$ condition that is not associated with increased morbidity or mortality. ${ }^{8,23,24}$

Mental status change was the symptom or sign most commonly documented in the 48 hours before this sample of $\mathrm{NH}$ residents had urine cultures obtained, and this observation has been noted previously regarding catheterized $\mathrm{NH}$ residents. ${ }^{12}$
The likely reason for obtaining cultures in these patients is concern that any change in cognition could represent incipient delirium, a common and dangerous condition in acutely ill hospital patients. ${ }^{25}$ However, most NH residents have dementia, which in the absence of acute illness is frequently associated with fluctuation in cognitive status, including affective, hyperactive, and delusional symptoms. ${ }^{26}$ Thus, although a recent systematic review found a modest association between delirium and urine infection in older persons, no study meeting review criteria was $\mathrm{NH}$ based, and all had methodological flaws. ${ }^{27}$ Therefore, little evidence supports ordering a urine culture when the only symptom is mental status change.

The second most common sign or symptom documented in these patients was a change in urine appearance. Alteration in urine color, odor, or sediment is associated with a positive urine culture, ${ }^{28}$ but the literature emphasizes that most people with cloudy or odorous urine are not sick..$^{29,30}$ Furthermore, bacteriuria in asymptomatic $\mathrm{NH}$ patients is 


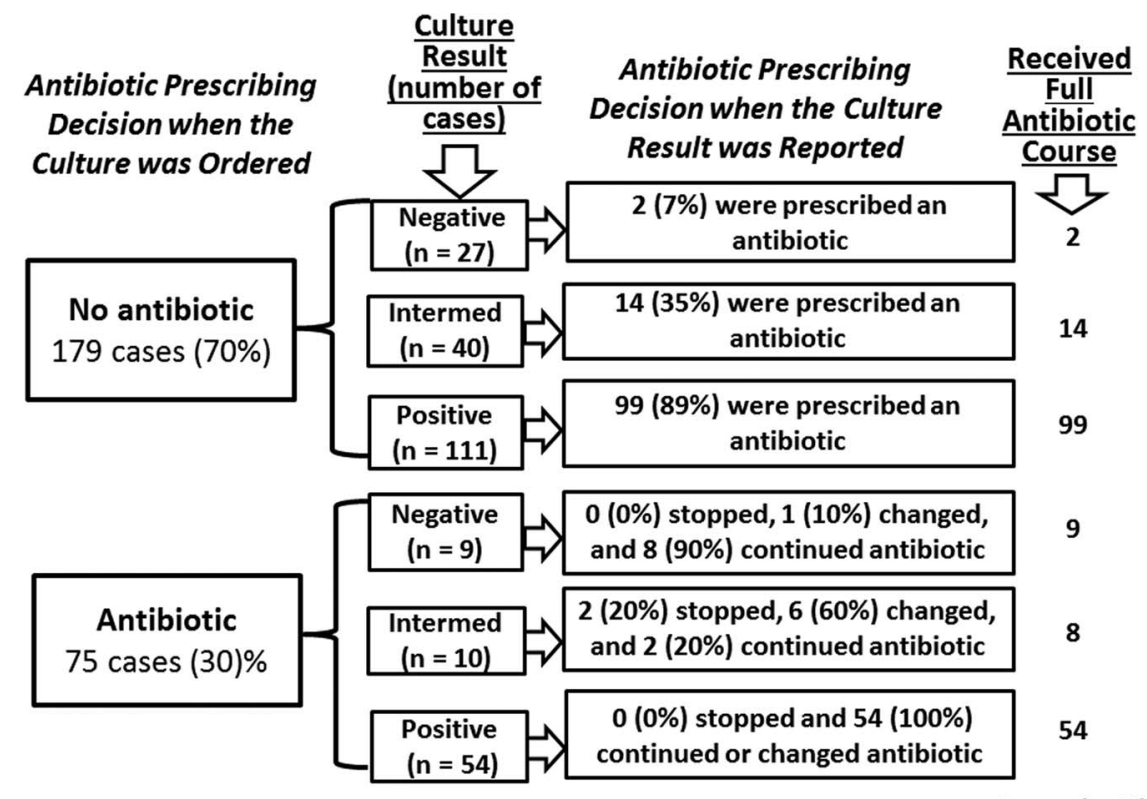

Total $186(74 \%)$

FIGURE 1. Flow chart demonstrating prescribing decisions among the 251 cases in which urine cultures and colony counts were ordered in 31 nursing homes (3 cases were excluded that reported organisms but no colony counts). While only $30 \%$ of the cases were associated with an initial antibiotic prescription, 74\% ultimately received a full course of antibiotics. Negative $=<10,000 \mathrm{CFU} / \mathrm{mL}$; Intermed (intermediate) $=$ 10,000-99,000 CFU/mL; Positive $=>100,000 \mathrm{CFU} / \mathrm{mL}$.

highly prevalent, and nonbacterial factors can alter the odor or appearance of voided urine. ${ }^{31,32}$ Subanalyses of these 43 cases verified this hypothesis; patients with changes in urine appearance did not differ significantly from the group overall: $12 \%$ had a temperature $\geq 37.2^{\circ} \mathrm{C}\left(99^{\circ} \mathrm{F}\right) ; 9 \%$ had dysuria documented; and $63 \%$ had no urinary tract-specific symptoms or signs documented. Thus, belief that a change in urine color, odor, or sediment indicates infection is likely to have influenced overprescribing of empirical antibiotics in our sample.

As these findings illustrate, addressing the complex interplay of factors that result in overprescribing for presumed UTIs will require multifaceted educational and quality improvement efforts. ${ }^{33}$ To date, the majority of antibiotic stewardship activities have involved guidelines aimed at reducing urine testing, with mixed results. ${ }^{22,34-36}$ Instead, we recommend that urinary-tract-oriented antibiotic stewardship include all of the following measures: (1) guidelines for urine testing; (2) tracking of urine culture results, and where volume of studies permits, creation of antibiograms to guide empirical treatment; (3) strict guidelines for what to do when urine culture results are reported; and (4) guidelines for initiating antibiotics, including recommended treatment duration. Observation ("watchful waiting") orders should also be implemented for use in patients with nonspecific or mild symptoms who do not meet guidelines for empirical antibiotics. These should include hydration; medication review; orientation procedures; measures to encourage restful sleep; evaluation for new or worsening cardiac, renal, or metabolic problems and for depressive symptoms; and regular documentation of symptoms and vital signs. ${ }^{6,37}$ Guidelines for responding to urine culture results should include an admonition to prescribe antibiotics only if the patient has clear signs of illness (eg, meets the modified McGeer criteria) ${ }^{17}$ plus discontinuation of antibiotics in persons with negative cultures. Guidelines should be endorsed by all medical providers and communicated clearly to all nursing staff, with room provided for discussion and protocol deviation in unusual situations.

\section{ACKNOWLEDGMENTS}

The following laboratories contributed urine culture results to one or more of our study nursing homes, which were included in our aggregate antibiogram: LabCorp, Solstas, Moore Regional Hospital, Betsy Johnson Regional Hospital, Columbus Regional Healthcare, Vidant Bertie Hospital, Halifax Regional Medical Center, Sampson Regional Medical Center, New Hanover Regional Medical Center, Meridian Laboratory, Pathologists Diagnostic Laboratory, NC Baptist Hospital, Johnston Medical Center, Rowan Medical Center, Lake Norman Regional Medical Center, Novant Health Medical Center, Caromont Regional Medical Center Laboratory, Wake Forest Baptist Health, Wayne Memorial Hospital, Harnett Health System Laboratory, and Gaston Memorial Hospital.

Financial support: This research was conducted by the Program on Aging, Disability, and Long-Term Care of the Cecil G. Sheps Center for Health Services Research at the University of North Carolina at Chapel Hill with support from the United States Agency for HealthCare Research and Quality (grant no. R18 HS022846-01).

Potential conflicts of interest: All authors report no conflicts of interest relevant to this article.

Address correspondence to Philip D. Sloane, 590 Manning Drive, Chapel Hill, NC 27599 (psloane@med.unc.edu). 


\section{REFERENCES}

1. Medicare and Medicaid programs: reform of requirements for long-term care facilities. Office of the Federal Register website. https://www.federalregister.gov/documents/2016/10/04/2016-23503/ medicare-and-medicaid-programs-reformof-requirements-for-longterm-care-facilities. Published 2016. Accessed November 7, 2016.

2. The core elements of antibiotic stewardship in nursing homes. Centers for Disease Control and Prevention website. http://www. cdc.gov/longtermcare/prevention/antibiotic-stewardship.html. Published 2015. Accessed August 9, 2016.

3. Daneman N, Gruneir A, Bronskill SE, et al. Prolonged antibiotic treatment in long-term care: role of the prescriber. JAMA Intern Med 2013;173:673-682.

4. Zimmerman S, Sloane P, Bertrand R, et al. Successfully reducing antibiotic prescribing in nursing homes. J Am Geriatr Soc 2014;62:907-912.

5. Eikelenboom-Boskamp A, Cox-Claessens J, Boom-Poels P, Drabbe M, Koopmans R, Voss A.. Three-year prevalence of healthcare-associated infections in Dutch nursing homes. J Hosp Infect 2011;78:59-62.

6. Nace D, Drinka P, Crnich C. Clinical uncertainties in the approach to long term care residents with possible urinary tract infection. J Am Med Dir Assoc 2014;15:133-139.

7. The national action plan to prevent health care-associated infections: road map to elimination; Chapter 8: Long-term care facilities. US Department of Health and Human Services website. http://health.gov/hcq/pdfs/hai-action-plan-ltcf.pdf. Published 2013. Accessed August 9, 2016.

8. Trautner B, Grigoryan L. Approach to a positive urine culture in a patient without urinary symptoms. Infect Dis Clin North Am 2014;28:15-31.

9. Phillips C, Adepoju O, Stone N, et al. Asymptomatic bacteriuria, antibiotic use, and suspected urinary tract infections in four nursing homes. BMC Geriatr 2012;12:73.

10. Nicolle L, Bradley S, Colgan R, Rice J, Schaeffer A, Hooton T. Infectious Diseases Society of America guidelines for the diagnosis and treatment of asymptomatic bacteriuria in adults. Clin Infect Dis 2005;40:643-654.

11. Cai T, Nesi G, Mazzoli S, et al. Asymptomatic bacteriuria treatment is associated with a higher prevalence of antibiotic resistant strains in women with urinary tract infections. Clin Infect Dis 2015;61:1655-1661.

12. Eke-Usim AC, Rogers MA, Gibson KE, Crnich C, Mody L. Targeted infection prevention study team. Constitutional symptoms trigger diagnostic testing before antibiotic prescribing in high-risk nursing home residents. J Am Geriatr Soc 2016;64: 1975-1980.

13. High K, Bradley S, Gravenstein S, et al. Clinical practice guideline for the evaluation of fever and infection in older adult residents of long-term care facilities: 2008 update by the Infectious Diseases Society of America. J Am Geriatr Soc 2009;57:375-394.

14. Loeb M, Bentley D, Bradley S, et al. Development of minimum criteria for the initiation of antibiotics in residents of long-termcare facilities: results of a consensus conference. Infect Control Hosp Epidemiol 2001;22:120-124.

15. Loeb M, Brazil K, Lohfeld L, et al. Optimizing antibiotics in residents of nursing homes: protocol of a randomized trial. $B M C$ Health Serv Res 2002;2:17.
16. Genao L, Buhr G. Urinary tract infections in older adults residing in long-term care facilities. Ann Long Term Care 2012;20: 33-38.

17. Stone N, Ashraf M, Calder J, et al. Surveillance definitions of infections in long-term care facilities: revisiting the McGeer criteria. Infect Control Hosp Epidemiol 2012;33:965-977.

18. Sloane PD, Kistler C, Mitchell CM, et al. Role of body temperature in diagnosing bacterial infection in nursing home residents. J Am Geriatr Soc 2014;62:135-140.

19. Fever temperatures: accuracy and comparison-topic overview. WebMD website. http://www.webmd.com/children/tc/fevertemperatures-accuracy-and-comparison-topic-overview. Published 2015. Accessed December 1, 2015.

20. Nursing Home Compare. US Government Medicare website. https://www.medicare.gov/NursingHomeCompare/search.html. Accessed August 31, 2015.

21. Rafii F, Sutherland J, Cerniglia C. Effects of treatment with antimicrobial agents on the human colonic microflora. Therapeut Clin Risk Manag 2008;4:1343-1358.

22. Loeb M, Brazil K, Lohfeld L, et al. Effect of a multifaceted intervention on number of antimicrobial prescriptions for suspected urinary tract infections in residents of nursing homes: cluster randomised controlled trial. BMJ 2005;331: 669-670.

23. Boscia J, Abrutyn E, Kaye D. Asymptomatic bacteriuria in elderly persons: treat or do not treat? Ann Intern Med 1987;106: 764-766.

24. Wagenlehner F, Naber K, Weidner W. Asymptomatic bacteriuria in elderly patients: significance and implications for treatment. Drugs Aging 2005;22:801-807.

25. Inouye S. Delirium in older persons. N Engl J Med 2006; 354:1157-1165.

26. Borsje P, Wetzels R, Lucassen P, Pot A, Koopmans R. The course of neuropsychiatric symptoms in community-dwelling patients with dementia: a systematic review. Int Psychogeriatr 2015;27: 385-405.

27. Balogun S, Philbrick J. Delirium, a symptom of UTI in the elderly: fact or fable? A systematic review. Canad Geriatr J 2013; 17:22-26.

28. Juthani-Mehta M, Quagliarello V, Perrelli E, Towle V, Van Ness $\mathrm{P}$, Tinetti M. Clinical features to identify urinary tract infection in nursing home residents: a cohort study. J Am Geriatr Soc 2009;57:963-970.

29. Midthun S, Paur R, Lindseth G. Urinary tract infections. Does the smell really tell? J Gerontol Nurs 2004;30:4-9.

30. Vaisman A, Gold W, Leis J. A 78-year-old woman with lethargy and a positive urine culture. CMAJ 2013;185:679-680.

31. Pandey S, Kim H, Choi S, Sa I, Oh S. Major odorants released as urinary volatiles by urinary incontinent patients. Sensors (Basel) 2013;13:8523-8533.

32. Nicolle L, Bradley S, Colgan R, Rice J, Schaeffer A, Hooton T. Infectious Diseases Society of America guidelines for the diagnosis and treatment of asymptomatic bacteriuria in adults. Clin Infect Dis 2005;40:643-654.

33. Wald H. Challenging the "Culture of Culturing": the case for less testing and more clinical assessment. JAMA Intern Med 2016;176:587-588.

34. Pettersson E, Vernby A, Mölstad S, Lundborg CS. Can a multifaceted educational intervention targeting both nurses and physicians change the prescribing of antibiotics to nursing home 
residents? A cluster randomized controlled trial. J Antimicrob Chemother 2011;66:2659-2666.

35. Trautner BW, Grigoryan L, Petersen NJ, Hysong S, Cadena J, Patterson JE, Naik AD. Effectiveness of an antimicrobial stewardship approach for urinary catheter-associated asymptomatic bacteriuria. JAMA Intern Med 2015;175:1120-1127.
36. Zabarsky TF, Sethi AK, Donskey CJ. Sustained reduction in inappropriate treatment of asymptomatic bacteriuria in a longterm care facility through an educational intervention. Am J Infect Control 2008;36:476-480.

37. Juthani-Mehta M, Datunashvili A, Tinetti M. Tests for urinary tract infection in nursing home residents. JAMA 2014;312:1687-1688. 